\title{
Reporte de caso clínico: Uso de TRAUMEEL y ZEEL T en combinación, para el manejo de osteoartrosis degenerativa de rodilla.
}

\author{
Jim roger Sánchez Benavidez ${ }^{1}$ \\ Autor para correspondencia: Jim roger Sánchez Benavidez, sjim041@gmail.com \\ 1 sjim041@gmail.com
}

\section{Resumen}

Informe de caso clínico de una paciente con antecedente de osteoartrosis degenerativa de rodillas, que le generaba bastante dolor y limitación para realizar actividades diarias como ; caminar, agacharse y subir escaleras. Durante años recibió manejo con medicina alopática sin un buen resultado.

Sometimos a la paciente a tratamiento con inyecciones semanales intraarticulares con una mezcla de traumeel y zeel $\mathrm{T}$, durante casi 10 meses, obteniendo resultados muy satisfactorios, con lo cual pudimos evidenciar que esta combinación es muy efectiva para producir regeneración del cartílago articular con la consiguiente regulación que produce mejoría de los síntomas relacionados a procesos inflamatorios degenerativos osteoarticulares, recuperación de la movilidad articular y control de edema y derrames articulares.

Palabras clave: Reporte de caso clínico, osteoartrosis, TRAUMEEL, ZEEL T

\section{Introducción}

No fue fácil encontrara literatura medica sobre estudios de casos clínicos similares de pacientes tratados con medicina integrativa biorreguladora de sistemas. El manejo que se le dio a la paciente con una mezcla de traumeel y zeel t intra-articular, fue basado en el ENSAYO CLINICO MOZART, de laboratorios HEEL.

El tratamiento se realizo con una frecuencia semanal, durante casi 10 meses, obteniendo una mejoría significativa en el control del dolor y la recuperación de la capacidad de movimiento de la articulación tratada.

(Un estudio doble ciego, aleatorizado, controlado con solución salina de la eficacia y seguridad de las inyecciones intraarticulares coadministradas de Tr14 y Ze14 para el tratamiento de la osteoartritis dolorosa de la rodilla: El ensayo MOZART,

Revista Europea de Medicina Integrativa, 
Volumen 13,2017,Páginas 54-63,

ISSN 1876-3820,

https://doi.org/10.1016/j.eujim.2017.07.005.

(https://www.sciencedirect.com/science/article/pii/S187638201730135X)

\title{
Cronología
}

\author{
2020-06-16 — la paciente presenta una calificación de womac inicial de 70/96 lo cual demuestra la \\ severidad del cuadro \\ $2020-12-15$ \\ se realiza cuestionario womac con una calificación de 45/96 que concuerda con la \\ evidencia clínica de mejoría dada por disminución de dolor, limitación y \\ recuperación de la movilidad articular \\ 2021-05-02 - Se aplica cuestionario womac evidenciando una calificación de 11/96 que concuerda \\ con la remisión de dolor, edema y mejoría de la movilidad en un altísimo porcentaje.
}

\section{Descripción del caso}

La paciente es una mujer de 61 años, maestra de profesión durante caso 40 años, trabajo en el cual según refiere ella estaba sometida a subir y bajar escaleras de forma rutinaria durante su jornada laboral, desde hace algo mas de 10 años empezó a notar dolor, inflamación y posterior limitación en sus rodillas, evaluada en servicio de salud con diagnostico de osteoartritis degenerativa de rodillas,

Estuvo bajo tratamiento alopático durante cuatro años con terapia farmacológica oral con aines y glucosamina - condroitina e infiltraciones ocasionales con cortico esteroides, sin mejoría notable; Por lo que se calificó como candidata a remplazo articular bilateral de rodillas, principalmente con mayor compromiso de rodilla derecha.

Antes de iniciar la terapia se realizo una medición de escala de severidad de dolor, rigidez y limitación de las articulaciones comprometidas, mediante la aplicación del cuestionario womac; inicialmente en junio de 2020, esta arroja un resultado de15/20 puntos para dolor; 7/8 puntos para rigidez y 48/68 puntos para limitación.

Inicialmente trazamos un plan de manejo con infiltraciones articulares con plasma auto- logó rico en plaquetas, con lo cual se evidencio por referencia de la paciente, mejoría del dolor, limitación y edema de las articulaciones, pero con efectos secundarios tempranos posteriores a la terapia referidos como desagradables para la paciente tales como; calor local, incremento del dolor y limitación que permanecían durante los primeros dos días después de la terapia con PRP. 
Teniendo en cuenta que esto generaba gran insatisfacción para la paciente, incluso llevándola a considerar abandonar el tratamiento; se decide cambiar la terapia e iniciamos la aplicación intraarticular con medicamentos bio-reguladores (TRAUMEEL, ZEELT).

El protocolo incluye la aplicación semanal intra articular en cada rodilla de TRAUMEEL 1 ampolla de 2,2 ml, ZEELT una ampolla de $2 \mathrm{ml}$, mezcladas en una jeringa de $5 \mathrm{cc}$, con aguja $26 \mathrm{G} \mathrm{x} \mathrm{1,5}$ pulgadas. Previa asepsia y antisepsia, con abordaje anterior- ventana interna.

Además terapia de soporte oral de TRAUMEEL, tres tabletas por día durante el primer mes de terapia.

A partir de la segunda sesión, la paciente empezó a percibir mejoría dada por disminución de signos locales de inflamación, dolor y limitación. Nunca refirió efectos secundarios locales ni sistémicos posteriores a la terapia.

Después de realizar de infiltraciones semanales durante un mes, se evidencia notable mejoría dada por aumento de la capacidad para realizar marcha y bipedestación por lapsos prolongados, disminución marcada del edema articular, desaparición de la crepitación presente al inicio del tratamiento, que incluso era evidente en el momento de la punción, al penetrar la aguja a través de la capsula articular; signo que en la actualidad ya no está presente.

También se evidencia mejoría en escala de puntuación de dolor (actualmente calificada por ella como de 1/10) y mejoría de la limitación que le permite actualmente realizar marcha y bipedestación prolongadas y otras actividades que para ella antes eran imposibles de realizar, tales como subir y bajar escaleras e incluso transitar por superficies irregulares, sin presentar dolor o sensación de inseguridad.

A todo lo anterior se le suma el beneficio de no presentar efectos secundarios a nivel articular, experimentados con otras terapias (aumento de dolor, inflamación, enrojecimiento y calor de la articulación durante los dos primeros días post terapia).

La aplicación final de la escala WOMAC arrojo una mejoría significativa dada por una calificación así para el 02 de julio de 2021. Dolor 3/20 puntos; Rigidez 0/8 y limitación 8/68.

Como dato adicional pudimos evidenciar que la paciente presentaba sobrepeso antes de iniciar la terapia, debido al sedentarismo generado por la imposibilidad de realizar actividad física por el dolor y la limitación en sus articulaciones. Como beneficio agregado, se dio de la recuperación de su capacidad de realizar actividades físicas, con lo cual refería sentirse más vital, pudiendo retomar actividades como marcha, subir y bajar escaleras, con lo cual logro una reducción de peso de aproximadamente $4 \mathrm{~kg}$.

\section{Punto de vista del paciente}

Antes de recibir tratamiento con la mezcla de traumeel y zeel t presentaba mucho dolor y limitación en las rodillas, lo cual me afectaba para realizar actividades del día a día como; subir o bajar escaleras, salir a caminar al mercado o agacharme a recoger algo del piso. 
Desde que inicie la terapia, a las pocas semanas comenzó el cambio; mejoro el dolor hasta desaparecer, la inflamación disminuyo marcadamente y lo mejor es que pude recuperar la movilidad de mis rodillas. Hasta el día de hoy no he vuelto a presentar dolor y puedo llevar una vida normal. Realmente fue una bendición de DIOS este tratamiento.

\section{Discusión}

La osteoartritis degenerativa es una enfermedad frecuente en los mayores de 50 años.

Es una patología compleja que involucra múltiples estructuras, de tejido conectivo articular y peri articular y su instauración es progresiva, mediada por factores mecánicos, bioquímicos que producen alteración en la función del condrocito y la matriz extracelular (MEC).

La terapéutica de base alopática es muy limitada, esta va encaminada solamente a controlar los síntomas, mejorar un poco la capacidad funcional articular y en última opción está el recambio articular protésico en casos avanzados y con una respuesta terapéutica pobre.

Los pacientes sometidos a terapias convencionales con AINES, cortico esteroides y suplementos de glucosamina y condroitina sulfato, pueden presentar mejoría de síntomas, pero con efectos deletéreos para la salud a largo plazo, en el caso del uso crónico de analgésicos, para nadie es un secreto que son gastro lesivos, pueden favorecer la aparición de nefritis y después de un largo periodo de uso ya no son efectivos para el control de dolor; y se inicia la carrera por aumentar la dosis y saltar de un grupo o marca comercial a otra, terminando por caer en el uso de opioides y opiáceos como estrategia final para el control del dolor, sin ningún beneficio evidente del tratamiento y más bien con muchos efectos secundarios nocivos para la salud.

Los medicamentos homeopáticos aparecen ahora, como una opción terapéutica que muestra gran efectividad en el control de síntomas y que genera una regulación orgánica de tal manera, que es evidente a la luz de los resultados, la recuperación de la biomecánica funcional articular.

TRAUMEEL es un compuesto que ha demostrado gran eficacia en el control de síntomas en patologías musculo esqueléticas agudas y también crónicas, gracias a componentes como el Aconitum napelus e Hypericum, controla dolor asociado a inflamación, el mercurius solubilis, atropa belladonna y hamamelis virginiana tienen acción anti-edema. La acción eficaz de TRAUMEEL se debe a la acción sinérgica de todos sus componentes. En especial la atropa belladona, ha mostrado un efecto en la producción de TGF-B, citokina con efecto inhibidor liberada por linfocitos como mensajero en la respuesta de ayuda de control inflamatorio, mediante el soporte inflamatorio dado por otras sustancias liberadas por linfocitos $\mathrm{T}$ específicamente como lo son el FNT e IL1.

Otra acción benéfica de este compuesto es que genera un efecto mediador de la respuesta celular, mediante la modulación de la secreción de IL 1B- IL 8 y FNT, por parte de monocitos, células T y células epiteliales gastrointestinales; además sin generar alteración en la adhesión plaquetaria ni producción de anión súper óxido (función normal de los granulocitos). 
Así mismo árnica montana tiene un aparente efecto inhibitorio selectivo sobre la producción del factor nuclear KB- que es un elemento regulatorio que promueve la transcripción de encimas antioxidantes como la súper oxido dismutasa, catalasa y glutatión, todas en función de control de la producción de ROS.

Ya está ampliamente demostrada la eficacia de ZEEL T como alternativa al uso de sustancias de inicio de acción lenta, que mejoren la viscosidad articular, como lo serian los productos derivados del ácido hialuronico intra articular y la suplementación oral con glucosamina y condroitina sulfato (Ensayo clínico MOZART).

Los neutrófilos son las células más abundantes, al igual que las citosinas pro inflamatorias, tales como IL-1 y TNF- $\alpha$. Se ha demostrado que las citosinas pro inflamatorias, IL-1, TNF- $\alpha$, IL-6 y otros integrantes de la súper familia de proteínas de IL6, tales como IL-7, IL-17 y IL-18, están asociados con las lesiones de cartílago y el desarrollo de OA.

El cartílago articular está compuesto por condrocitos y matriz extracelular, donde una red de colágeno es responsible de la resistencia a la tracción de la matriz, y los proteoglicanos son responsables del edema osmótico y de las propiedades elásticas del tejido cartilaginoso.

En las articulaciones con osteoartritis, la matriz extracelular del cartílago se degenera y sufre alteraciones bioquímicas y estructurales, seguida por su degradación y fallo progresivo de sus propiedades biomecánicas.

A medida que el cartílago se degenera, se deteriora su capacidad funcional. Si bien la matriz extracelular es la unidad funcional en este proceso, los condrocitos también tiene un papel preponderante en la homeostasis articular.

Las citosinas inflamatorias y los productos de degradación de la matriz estimulan a las metaloproteínasas de la matriz (MMP) para inducir la degradación de tejidos viejos o dañados. Lo anterior contrarrestado por una serie de factores de crecimiento, como el TGF- $\beta$, las Proteínas Morfogenéticas Óseas (BMP), que inhiben las acciones de las MMP, y por lo tanto, inducen reparación de los tejidos. Esta oscilación catabólica/anabólica es de suma importancia para mantener la integridad de los tejidos sanos.

Cuando se altera este proceso debido al daño tisular, fuerzas mecánicas, o cuando se reduce la capacidad de iniciar la reparación a causa de déficit en los factores de crecimiento o la incapacidad para responder a ellos, lo cual se observa con mayor frecuencia en la senilidad, se produce un ciclo catabólico/anabólico super activo.

Para comprender mejor la afección del cartílago, al menos cuando está mediado por los condrocitos (condrólisis condrocítica), es necesario estudiar los mecanismos moleculares que alteran el equilibrio entre la actividad catabólica y anabólica de los condrocitos.

Dado que los condrocitos sufren muerte celular en algún momento del proceso de destrucción del cartílago, también es importante saber si estos factores moleculares también contribuyen a la muerte 
celular.

La mitocondria, tiene un papel fundamental, ya que una producción adecuada de ATP, permite al condrocito mantener una adecuada homeostasis. Los procesos de OA, se caracterizan por una alteración mitocondrial, con una baja producción energética, lo que acelera el proceso de la apoptosis condrocitaria

La causa de la sinovitis crónica en la OA no se comprende plenamente. Pueden hallarse restos o partes del cartílago en la membrana sinovial, donde provocan las respuestas típicas a cuerpos extraños.

La lesión mecánica del cartílago también puede llevar a la producción de radicales libres, incluyendo especies reactivas de oxígeno (ROS).

El factor de crecimiento transformante beta (TGF- $\beta$, ) es una proteína que controla la proliferación y la diferenciación celular. De hecho, la síntesis de TGF- $\beta$ es uno de los mecanismos más importantes para controlar las reacciones inflamatorias en los tejidos debido a su inhibición de las células proinflamatorias del sistema inmune.

Se ha observado que en la OA, el cartílago presenta una reducción en su elasticidad y fuerza, junto con debilitamiento, ruptura fibrilar y ulceración. También disminuye la elasticidad del colágeno, lo que está asociado con una menor capacidad por parte del cartílago de resistencia en el movimiento.

Los cambios en la visco elasticidad del cartílago son evidentes muy temprano en el proceso dela osteoartritis.

El WHE 1990; es un estudio in vitro que sugiere que el tratamiento con Zeel T produce un aumento en la elasticidad del cartílago en la osteoartritis, mejorando sus propiedades biomecánicas.

Podemos observar que el tratamiento con medicamentos biorreguladores es efectivo y no tiene efectos secundarios que generen mayor disconfort para los pacientes; es decir, tienen un perfil de bioseguridad muy alto.

Pudimos evidenciar la efectividad de estos medicamentos homotoxicologicos durante el transcurso del tratamiento, mediante la medición de síntomas por medio de la aplicación del cuestionario WOMAC, determinando una puntuación clara y evidente de la mejoría, desde la evidencia de la recuperación de la funcionalidad de las articulaciones comprometidas, además de los cambios radiológicos evidenciados en la comparación de Rx realizados en 2016 versus los Rx de control de mayo de 2021

\section{Conclusión}

La evidencia científica encontrada en estudios clínicos que demuestran la efectividad del tratamiento con terapias no farmacológicas, está en concordancia con lo que se puede ver en la evolución clínica favorable de los pacientes tratados con TRAUMEEL y ZEELT intra-articular; mostrando una disminución marcada de síntomas como dolor, edema, rigidez y limitación de las articulaciones tratadas. 
El tiempo de respuesta a la terapia, en la cual la paciente refirió sentir y notar mejoría, fue a posterior a la segunda sesión de infiltraciones.

Este tipo de terapia es muy efectiva incluso como opción terapéutica única, ya que la paciente en ningún momento durante o después del tratamiento, tuvo necesidad de recurrir al uso de AINES u otro tipo de analgésico o anti-inflamatorio, debido a la remisión completa de los síntomas relacionados al proceso inflamatorio y degenerativo articular.

Pudimos evidenciar la mejoría clínica sustentada por lo s resultados de la aplicación de el cuestionario WOMAC al inicio de la terapia, 6meses después y a los 10 meses de tratamiento.

Esta terapia es muy segura y el procedimiento es muy bien tolerado por la paciente, ya que durante todo el tratamiento, no presento ningún efecto secundario o complicación derivada del mismo o de los compuestos utilizados; incluso nunca presento signos de inflamación local en la articulación o en el sitio de la punción y mucho menos a nivel sistémico durante o después de cada aplicación.

La paciente después de cada sesión, seguía juiciosamente las recomendaciones de guardar reposo y evitar actividades de esfuerzo tales como; caminatas largas, levantar cargas o subir y bajar escaleras; durante 12-24 horas, con el fin evitar así cualquier complicación.

Refirió siempre que al siguiente día, ya podía retomar sus actividades diarias sin inconveniente y nunca presento complicaciones tempranas ni tardías.

A la fecha, seguimos el mismo protocolo de manejo, con una regularidad de una sesión al mes en cada rodilla.

La paciente no ha presentado recaídas, niega reaparición de síntomas, incluso refiere remisión total del dolor y no ha presentado deterioro de su capacidad funcional articular.

Los resultados de este caso apoyan el uso de este par de compuestos simultáneamente, como una opción terapéutica adecuada para pacientes con artrosis de rodilla, que no responden a los tratamientos convencionales para manejo del dolor o que están en grupo de riesgo y su última opción terapéutica es el remplazo articular.

\section{Agradecimientos}

Inicialmente a Dios padre por permitirme conocer de las bondades de la medicina integrativa biorreguladora de sistemas.

A la paciente que amablemente me permitió exponer su caso y que me brindo la confianza de poder ayudarla en el proceso terapéutico frente a su enfermedad.

A mi familia por creer en mis capacidades y por su apoyo constante e incondicional.

A mis docentes; el Dr. Baltazar guerrero por estar acompañándome en este proceso y al Dr. Ralfo Santamaria por los aportes de su larga experiencia en el uso de la medicina integrativa biorreguladora en el área de la ortopedia. 


\section{$\underline{\text { Referencias }}$}

Arthritis Care Res. (Hoboken), 64 ( 4 (abril) ) ( 2012 ) , págs. 465 - 474

CrossRefVer registro en ScopusGoogle Académico

Academia Estadounidense de Cirujanos Ortopédicos

Tratamiento de la osteoartritis de rodilla.

Directriz basada en evidencia (segunda edición), AAOS (2013)

Google Académico

Instituto Nacional de Excelencia en Salud y Atención (Reino Unido)

Osteoartritis: cuidado y manejo

NICE Clinical Guideline No. 177 , Instituto Nacional para la Excelencia en la Salud y la Atención, Reino Unido ( 2014 ), p. 2014

Google Académico

J.-Y. Reginster, C. Cooper, M. Hochberg, J.-P. Pelletier, R. Rizzoli , J. Kanis , et al.

Comentarios sobre las recomendaciones discordantes para el uso de fármacos sintomáticos de acción lenta en la osteoartritis de rodilla

Curr. Medicina. Res. Opin. , 31 ( 5 ) ( 2015 ), pp. 1.041 mil - 1,045

CrossRefVer registro en ScopusGoogle Académico

O. Bruyère, C. Cooper, JP Pelletier, J. Branco, M. Luisa Brandi, F. Guillemin, et al.

Una recomendación de algoritmo para el tratamiento de la osteoartritis de rodilla en Europa e internacionalmente: un informe de un grupo de trabajo de la Sociedad Europea para los Aspectos Clínicos y Económicos de la Osteoporosis y la Osteoartritis (ESCEO)

Semin. Artritis Rheum., Elsevier, 44 ( 3 ) ( 2014 ), págs. 253 - 263

Kolhe, R., Hunter, M., Liu, S. et al. Expresión diferencial específica de género de miARN exosomal en líquido sinovial de pacientes con osteoartritis. Sci Rep 7, 2029 (2017). https://doi.org/10.1038/s41598-017-01905-y

Revista de la Facultad de Medicina

Versión impresa ISSN 0120-0011

rev.fac.med. vol.56 no.2 Bogotá Abr./Junio 2008

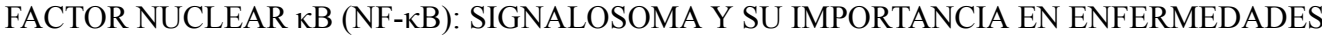 INFLAMATORIAS Y CÁNCER}

Nuclear factor kB (NF-KB): signalosoma and its importance in cáncer and inflammatories diseases 
Nancy P. Echeverri R1, Ismena Mockus S.2,

1. Estudiante Maestría en Ciencias-Bioquímica, Departamento de Química, Facultad de Ciencias, Universidad Nacional de Colombia, Bogotá

Correspondencia: npecheverrir@unal.edu.co

2. Docente, Departamento de Ciencias Fisiológicas, Facultad de Medicina, Universidad Nacional de Colombia, Bogotá.

Lancet (Londres, Inglaterra), 386 ( 9991 (25 de julio) ) ( 2015 ), págs. 376 - 387

ArtículoDescargar PDF Ver registro en ScopusGoogle Académico

Rev Col Med Fis Rehab 2017; 27(2):160-184 - http://revistacmfr.org

DOI: $10.28957 /$ rcmfr.v27n2a4

Recomendaciones sobre diagnóstico, Prevención y tratamiento farmacológico y no

Farmacológico de la osteoartritis (OA) de Rodilla

Monique Chalem Choueka1, César Ernesto Arango Pilonieta2, Mario Enrique Díaz Cortes3, John Duperly

Sánchez4, Juan Pablo Forero Franco5, Paulo José Llinás Hernández 6, Carlos Eduardo Rangel Galvis7, Carlos Rodríguez Martínez8, Federico Rondón Herrera9, Diego Velásquez Ramírez10

Carlos J. Lozada, Eve del Rio, Donald P. Reitberg, Robert A. Smith, Charles B. Kahn, Roland W. Moskowitz, A double-blind, randomized, saline-controlled study of the efficacy and safety of co-administered intra-articular injections of Tr14 and Ze14 for treatment of painful osteoarthritis of the knee: The MOZArT trial,

Revista Europea de Medicina Integrativa,

Volumen 13,2017,Páginas 54-63,

ISSN 1876-3820,

https://doi.org/10.1016/j.eujim.2017.07.005.

(https://www.sciencedirect.com/science/article/pii/S187638201730135X)

American college of reumathology.

Meeting abstracts.

Numero de resumen: 2896

Un estudio multicéntrico doble ciego, aleatorizado, controlado aleatorio (db-ECA) para evaluar la eficacia y seguridad de coadministrado Traumeel ${ }^{\circledR}\left(\right.$ TR14) y Zeel ${ }^{\circledR}($ Ze14) intraarticular (IA) Las inyecciones Versus IA placebo en pacientes con dolor moderado a severo asociado con OA de la rodilla

Reunión anual 2014 ACR / ARHP 


\section{NATIONAL LIBRARY OF MEDICINE}

National center of biotechnology information.

Schneider C. Traumeel - an emerging option to nonsteroidal anti-inflammatory drugs in the management of acute musculoskeletal injuries. Int J Gen Med. 2011 Mar 25;4:225-34. doi: 10.2147/IJGM.S16709. PMID: 21556350;

PMCID: PMC3085232

Artículo gratuito de PMC

Monografía ZeelT

Primera publicación en inglés, 2013 Primera Edición en español, 2015 Actualizada según la Segunda Edición en inglés, de junio de 2015 C 2015 by Biologische Heilmittel Heel GmbH, Alemania 79216 15/06. Dr. ReckewegStraße 2-4, 76532 Baden-Baden, Alemania.

Monografía Traumeel

Primera publicación en inglés, 2009 Tercera Edición en español 2015 Actualizada según la Sexta Edición en inglés, de junio de 2015 (C) 2015 by Biologische Heilmittel Heel GmbH, Alemania 71245 15/06 Biologische Heilmittel Heel GmbH Dr. Reckeweg-Straße 2-4, 76532 Baden-Baden, Alemania.

\section{Archivos adjuntos}




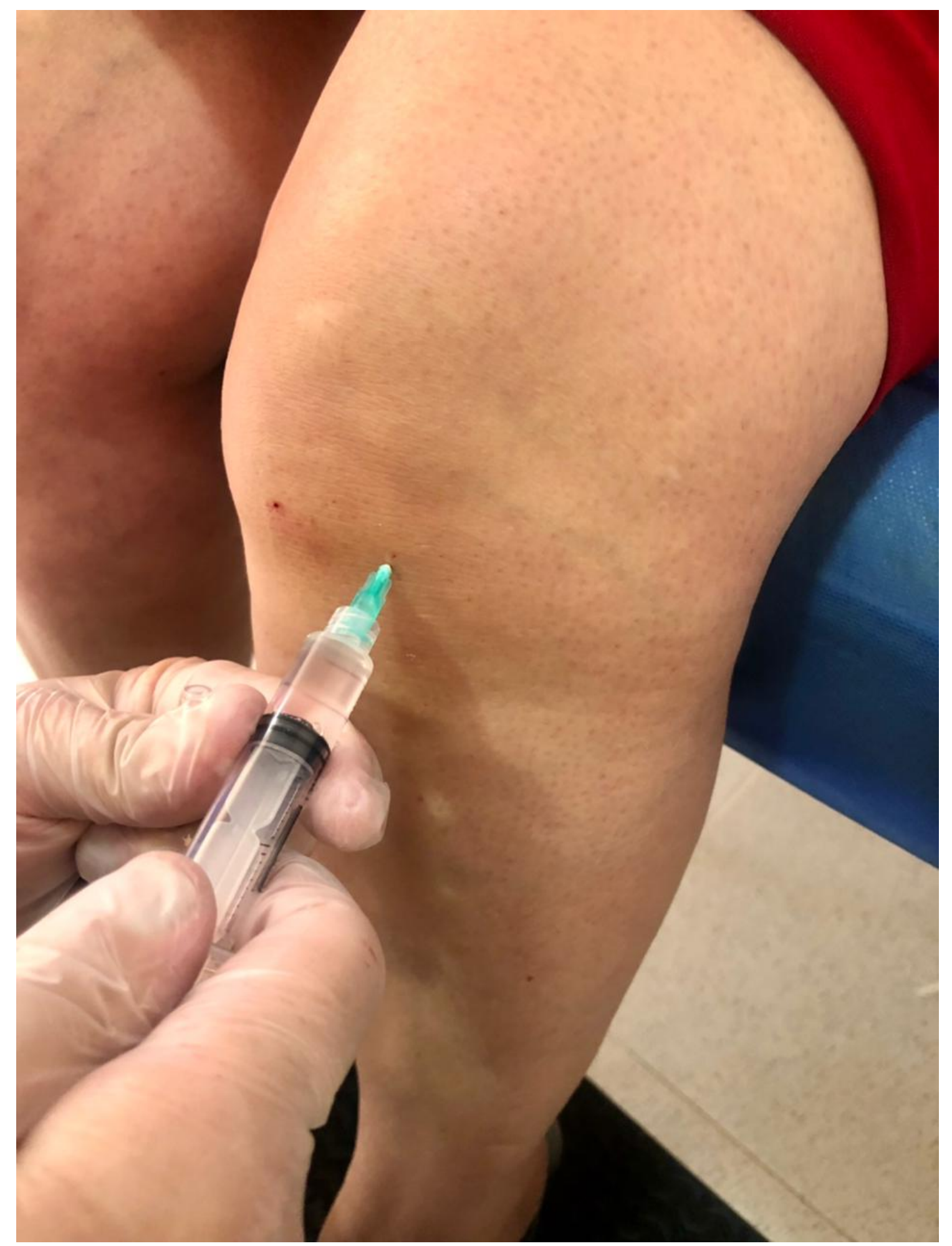

Técnica de infiltración articular de rodilla. Aplicación de mezcla de Traumeel + Zeel T. 


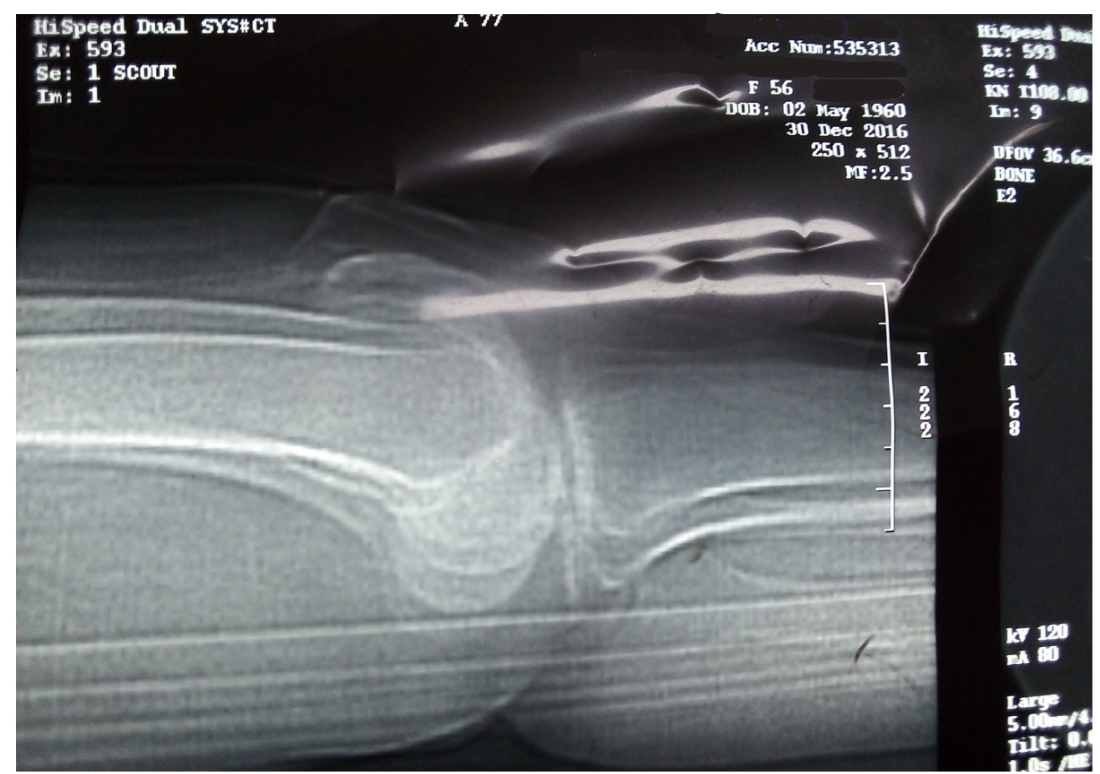

$R x$ de rodilla del 30 de diciembre de 2016. Se pueden evidenciar cambios severos, relacionados con artrosis. 


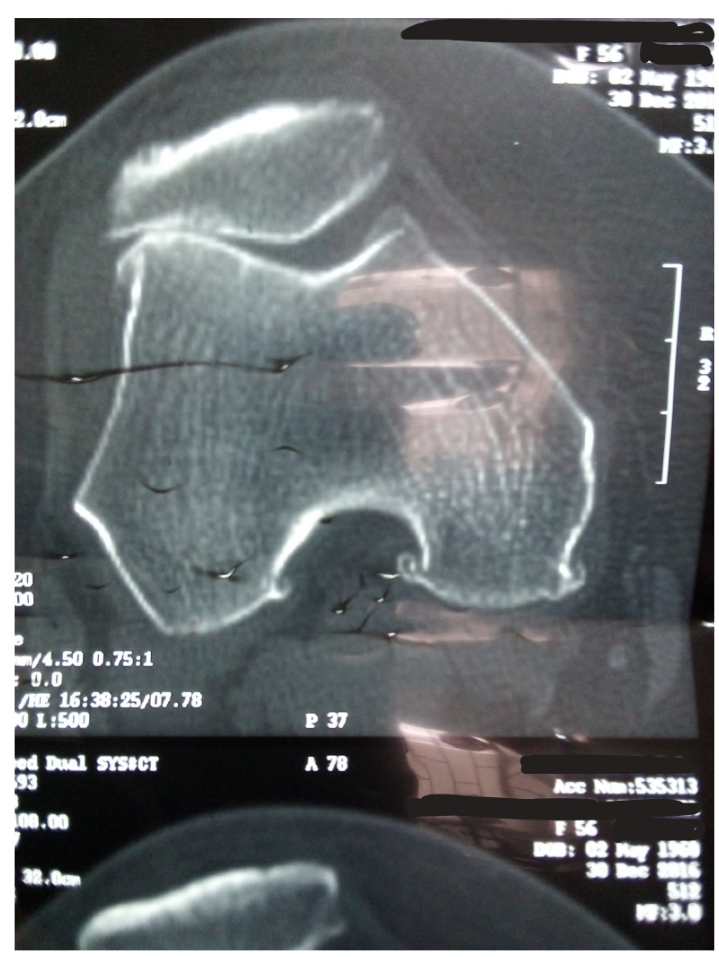

Se puede observar disminución del espacio pre patelar e irregularidades sobre la superficies articulares, además de presencia de osteofitos. 


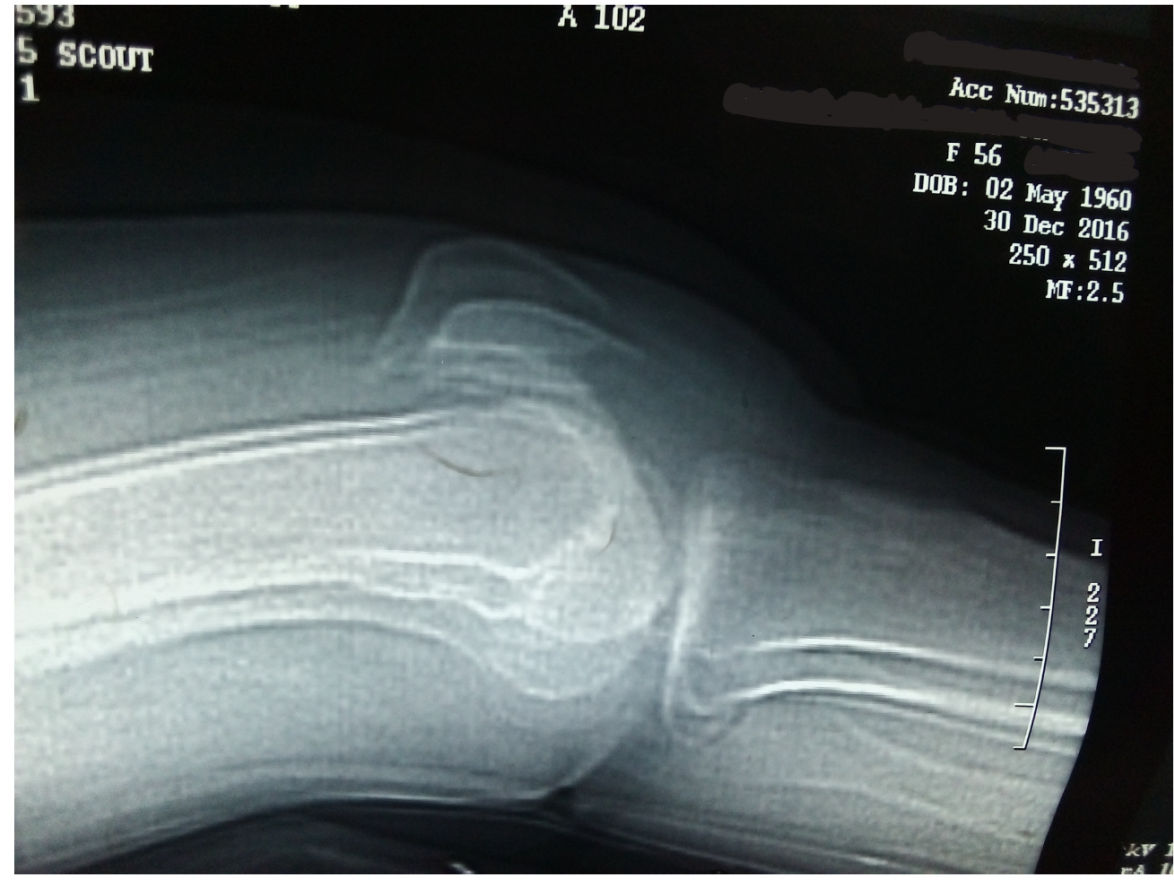

$R x$ de rodilla del 30 de diciembre de 2016. 


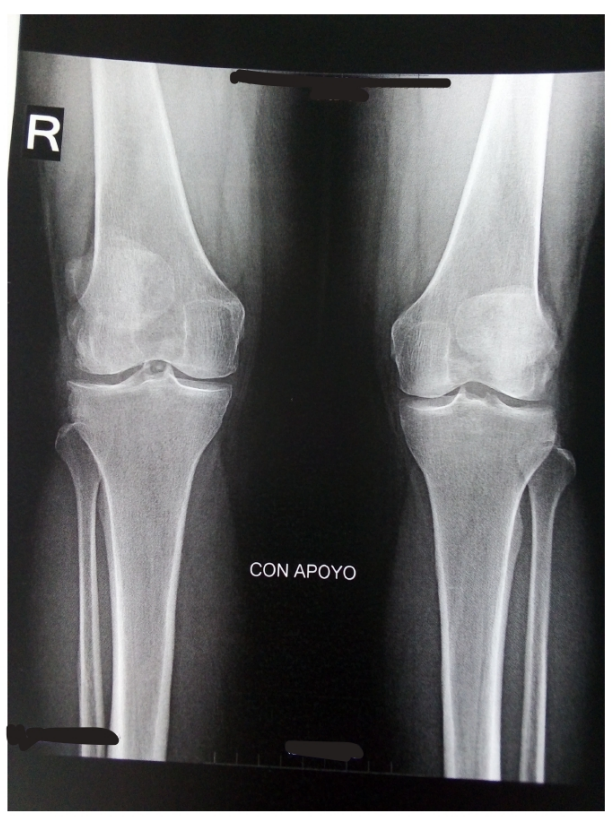

$R X$ de rodilla posterior a tratamiento (mayo de 2021). podemos observar recuperación del espacio articular, signos de regeneración del cartílago articular. 


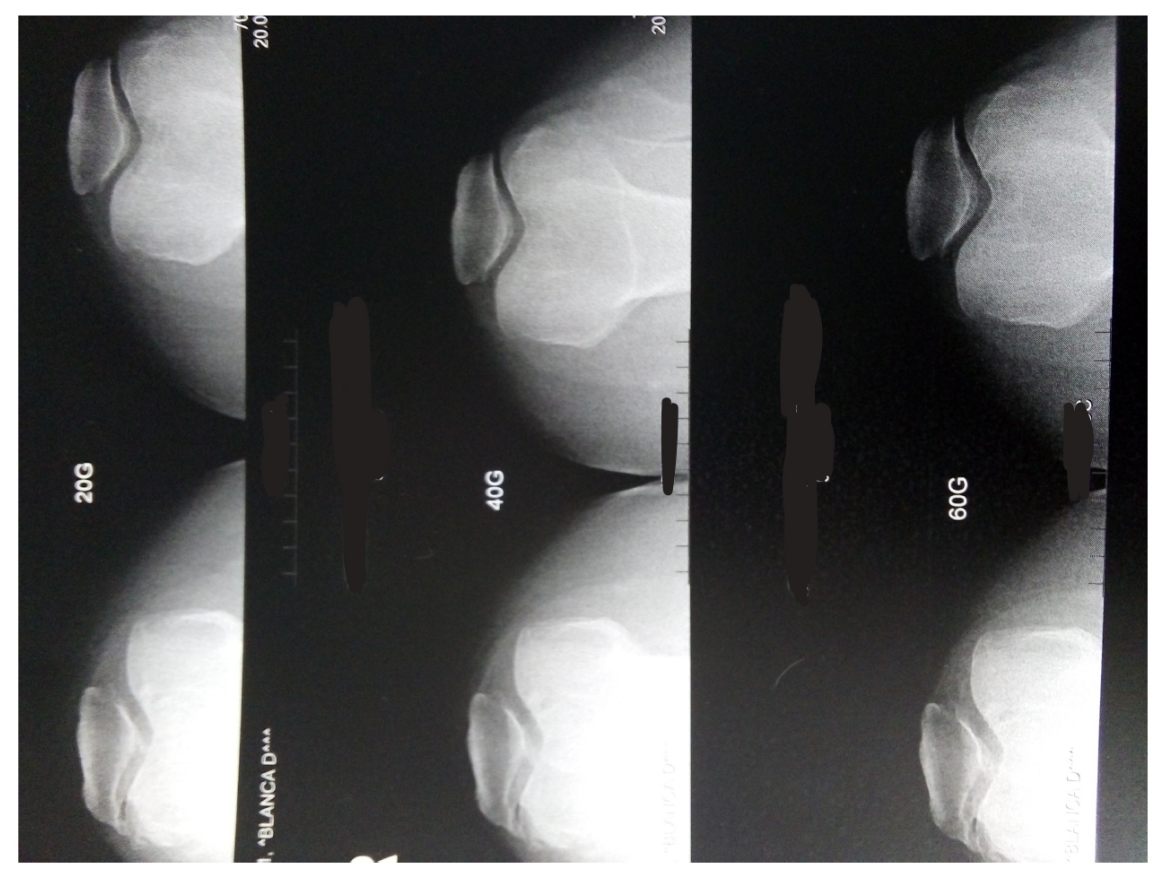

$R x$ de rodilla posterior a tratamiento. (mayo 30 de 2021). se pude evidenciar alineación patelo-femoral, recuperación de espacio retro-patelar. signos de regeneración del cartílago articular. 


\begin{tabular}{|c|c|c|c|}
\hline \multicolumn{2}{|l|}{ \#\#\# C A $\mathrm{ARE}$} & \multicolumn{2}{|l|}{ CARE Checklist of information to include when writing a case report } \\
\hline Topic & Item & Checklist item description & Reported on Line \\
\hline Title & 1 & The diagnosis or intervention of primary focus followed by the words "case report". & y \\
\hline Key Words & 2 & 2 to 5 key words that identify diagnoses or interventions in this case report, including "case report" & y \\
\hline \multirow{4}{*}{$\begin{array}{l}\text { Abstract } \\
\text { (no references) }\end{array}$} & 3a & Introduction: What is unique about this case and what does it add to the scientific literature? ...... & $\mathrm{y}$ \\
\hline & $3 b$ & Main symptoms and/or important clinical findings .......... & $\mathrm{y}$ \\
\hline & $3 c$ & The main diagnoses, therapeutic interventions, and outcomes . & $\mathrm{y}$ \\
\hline & $3 d$ & Conclusion —What is the main "take-away" lesson(s) from this case? . . . . . . . . . . . . . . . & $\mathrm{y}$ \\
\hline Introduction & 4 & One or two paragraphs summarizing why this case is unique (may include references) $\ldots \ldots \ldots \ldots$. & $y$ \\
\hline \multirow[t]{4}{*}{ Patient Information } & $5 a$ & De-identified patient specific information. . . . . . . & y \\
\hline & $5 b$ &  & $y$ \\
\hline & $5 c$ & Medical, family, and psycho-social history including relevant genetic information ...... & y \\
\hline & $5 d$ & Relevant past interventions with outcomes ........... & $\mathrm{y}$ \\
\hline Clinical Findings & 6 & Describe significant physical examination (PE) and important clinical findings. . & y \\
\hline Timeline & 7 & Historical and current information from this episode of care organized as a timeline & y \\
\hline \multirow{4}{*}{$\begin{array}{l}\text { Diagnostic } \\
\text { Assessment }\end{array}$} & $8 a$ & Diagnostic testing (such as PE, laboratory testing, imaging, surveys)........... & y \\
\hline & $8 b$ & Diagnostic challenges (such as access to testing, financial, or cultural) ............. & $\mathrm{y}$ \\
\hline & $8 c$ & Diagnosis (including other diagnoses considered) $\ldots \ldots \ldots \ldots \ldots$ & y \\
\hline & $8 d$ & Prognosis (such as staging in oncology) where applicable . & y \\
\hline \multirow{3}{*}{$\begin{array}{l}\text { Therapeutic } \\
\text { Intervention }\end{array}$} & 9a & Types of therapeutic intervention (such as pharmacologic, surgical, preventive, self-care) $\ldots \ldots \ldots \ldots \ldots \ldots$ & y \\
\hline & $9 b$ & Administration of therapeutic intervention (such as dosage, strength, duration). & $y$ \\
\hline & $9 c$ & Changes in therapeutic intervention (with rationale) $\ldots \ldots \ldots \ldots \ldots \ldots \ldots$ & y \\
\hline \multirow{4}{*}{$\begin{array}{l}\text { Follow-up and } \\
\text { Outcomes }\end{array}$} & $10 \mathrm{a}$ & Clinician and patient-assessed outcomes (if available) . & y \\
\hline & $10 \mathrm{~b}$ & 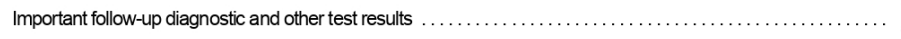 & $\mathrm{y}$ \\
\hline & $10 \mathrm{c}$ & Intervention adherence and tolerability (How was this assessed?) . . . . . . . . . . . . . . . . & y \\
\hline & 10d & Adverse and unanticipated events . & y \\
\hline \multirow[t]{4}{*}{ Discussion } & $11 \mathrm{a}$ & A scientific discussion of the strengths AND limitations associated with this case report... & $\mathrm{y}$ \\
\hline & 11b & Discussion of the relevant medical literature with references. ................... & y \\
\hline & $11 \mathrm{c}$ & The scientific rationale for any conclusions (including assessment of possible causes). & 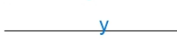 \\
\hline & 11d & The primary "take-away" lessons of this case report (without references) in a one paragraph conclusion ...... & y \\
\hline Patient Perspective & 12 & The patient should share their perspective in one to two paragraphs on the treatment(s) they received. ....... & y \\
\hline Informed Consent & 13 & Did the patient give informed consent? Please provide if requested & Yes $\square$ No \\
\hline
\end{tabular}


Este formulario está redactado conforme al Discussion document on Best Proctice for Consent for Publishing Medical Case Reports de COPE (Committee On Publication Ethics), disponible en uwww.publicationethics.ore publicado el 25 de febrero de 2016, acceso el 31 de julio de 2020

\section{Consentimiento Informado del Paciente del Caso Clínico}

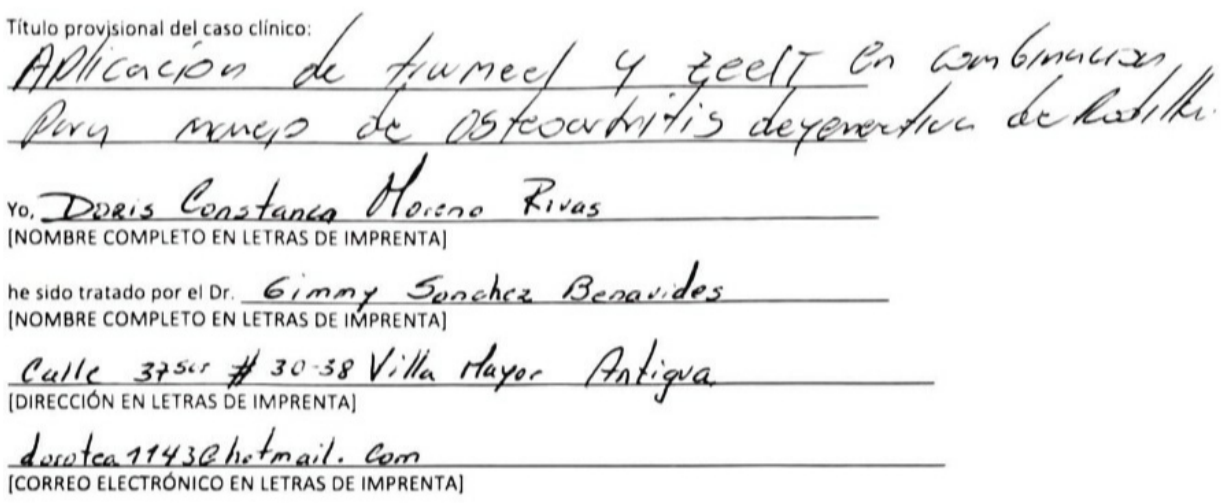

Por la presente confirmo que yo: (por favor, marque la casilla para confirmar)

\section{estoy legalmente autorizado para dar este consentimiento}

Mediante la firma de este formulario de consentimiento autorizo a este médico y a otros coautores a escribir un caso clínico sobre mi tratamiento. Entiendo que:

No voy a obtener ningún beneficio directo de la participación en este caso clínico. No obstante, la información que puede compartirse con otros profesionales sanitarios puede mejorar la atención de otras personas en el futuro.

La firma de este formulario de consentimiento no anula mis derechos de privacidad. Mi nombre y mi información médica protegida (IMP) no se compartirán salvo que se solicite y consienta mi autoría. No obstante, entiendo que no es posible garantizar un anonimato total. Es posible que alguien en algún lugar, por ejemplo, alguien que me haya cuidado o un familiar, me reconozca.

La información que se usa para este caso clínico incluye la anamnesis de mi enfermedad, relevante para este caso, los hallazgos de laboratorio, las fotografias y los protocolos de los tratamientos recetados.

Se presentará un resumen de mi tratamiento para su evaluación por un panel de expertos e investigadores, y se publicará en una revista médica con licencia de acceso libre (quien quiera puede consultar y leer libremente la publicación en línea), y/o se presentará en una conferencia médica. Doy mi consentimiento para dicha publicación o presentación. El resumen de mi tratamiento y el caso clínico no contendrá ningún dato personal. En el caso de que se tenga que incluir algún dato de carácter personal, este se publicará de manera anonimizada.

La autorización a usar mi información en este caso clínico no me supondrá costes adicionales. No recibiré ningún tipo de compensación. 


\section{Case Report Authorship and Copyright Consent}

I confirm that I am the primary and corresponding author of the submitted clinical case report. I confirm that all co-authors have given their consent and permission for submission of this clinical case report and I am authorized to grant the rights mentioned below to Heel without infringement of any third parties' rights.

I confirm that I anonymized the submitted clinical case report according to national and international data protection laws, and the patient cannot be recognized as a person through publication of this case report. I confirm that I have obtained the written Patient Information Consent in the form provided by Award organizers.

I will inform Biologische Heilmittel Heel GmbH ("Heel") immediately in writing after the patient has withdrawn his/her consent to participate in the clinical case report.

Heel is entitled to inform the press about the presentation of the award, the award winner(s) and all clinical case studies submitted for the award.

Signature of the submitting (corresponding) author
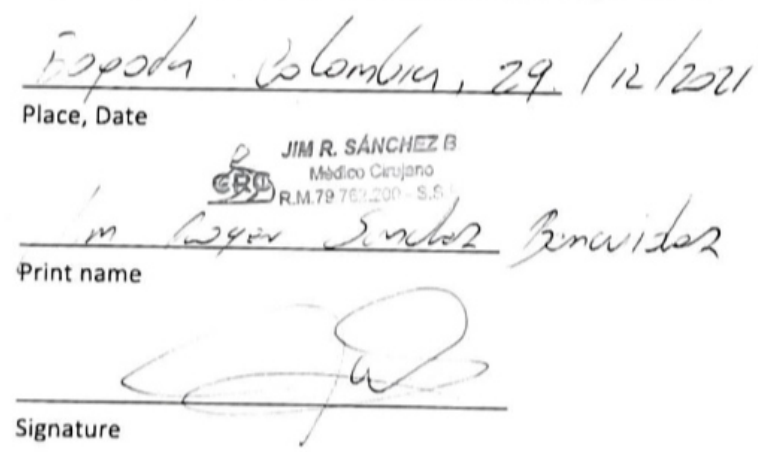
Este formulario está redactado conforme al Discussion document an Best Proctice for Consemt for Publisting Medical Case Reports de COPE (Committer On Publication Ethiss). disponible en wrmw. pablicationethics.ore publicado el 25 de febrero de 2016, acceso el 31 de fulio de 2020

Mi participación es totalmente voluntaria, y puedo revocar mi permiso para participar en este caso clínico en cualquier momento Todos mis datos personales enviados a Heel se eliminarán después de recibir la notificación del médico encargado del tratamiento sin embargo, una ver que el caso clinico se haya escrito y publicado (con los datos personales anonimizados) no podré retirarme Mi decisión no supondra una sanción ni una pérdida de los beneficios a los que tenga derecho como la calidad de la atención que reciba. La retirada puede enviarse al médico del tratamiento (consulte los datos de contacto arriba).

Me pueden pedir que contribuya con una aperspectiva del pacienten sobre el tratamiento que he recibido Puedo decidir voluntariamente proporcionar esta perspectiva o declinar

Mediante la firma de este formulario confirmo que:

- Me he declarado conforme a participar en este caso clínico

- Autorizo el acceso a la información personal de mi salud (historia clínica) como se explica en este formulario

- Me han informado de que no estoy obligado a participar en este caso clínico

- Se me ha informado acerca de los riesgos y los beneficios, si los hubiera, de permitir usar mi información en este caso clínico

- He leido todas las páginas de este formulario

- He visto una versión de un caso clínico a enviar o publicar (incluidas las fotografias) y me declaro conforme

- Me han explicado el reporte del caso clínico por completo y todas mis preguntas se han contestado a mi satisfacción

A firmar por el paciente o por el cuidador legaimente autorizado

$$
\begin{aligned}
& \text { Fecha } 2901</ 2021 \\
& \text { Lugar Bigota/rolenbis } \\
& \text { Dombre en ietras de imprenta Canstraconturno Ruers } \\
& \text { Firme Dom C. Horne } \mathrm{E} \text {. }
\end{aligned}
$$

A firmar por el médico del tratamiento, que ha explicado y entregodo el formulario al paciente
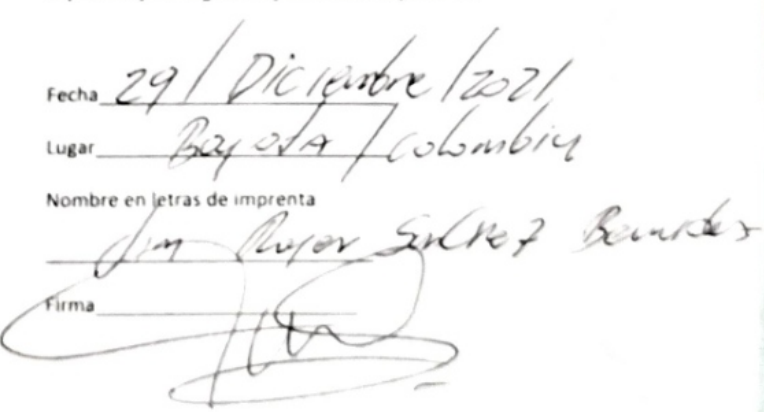